\title{
The enumeration and bifurcations of ranking functions
}

\begin{abstract}
W.J. Walker
Suppose $n$ competitors each compete in $r$ races and a ranking function $F$ assigns a score $F(j)$ to the competitor finishing in the $j$ th position in each race. The sum of the scores over $r$ races gives each competitor a final ranking. If $n$ is fixed, the ranking function $F$ bifurcates as $r$ increases. The complete bifurcation behaviour is determined for $n=3$ and some information obtained for $n>3$.
\end{abstract}

\section{Introduction}

A ranking function is used to give an overall ranking to $n$ competitors who compete in a sequence of $r$ races. We define a "ranking function $F "$ to be a nonnegative function defined on the first $n$ positive integers and satisfying the condition $F(j)>F(j+1)$, for $1 \leq j \leq n-1$. In each race the competitor finishing in the $j$ th position is awarded a score $F(j)$. The sum of the scores over the $r$ races gives each competitor a final score and the competitors are ranked by these final scores.

A "result" will be simply a finite set of positive integers $\left\{\alpha_{k}\right\}_{1 \leq k \leq r}$, where for each $k, I \leq \alpha_{k} \leq n$. That is, a result represents the placings of a single competitor over the $r$ races. (We do not allow the possibility that two competitors be placed equal in a given race.)

Two ranking functions are said to be " $n: r$ equivalent" if for any set of results for $n$ competitors in $r$ races they give the same final

Received 20 January 1978. 
rankings. Clearly $n: r$ equivalence is an equivalence relation in the usual sense.

EXAMPLE 1.1. Suppose $F$ is a ranking function for some fixed $n$ and $r$ and that $m$ and $c$ are positive constants. Then an $n: r$ equivalent ranking function $E$ may be defined by setting

$$
E(j)=m F(j)+c \text { for } 1 \leq j \leq n \text {. }
$$

EXAMPLE 1.2. Suppose $n=3, r=2$, and $F, G, H$ are defined such that

$$
\begin{aligned}
& F(1)+F(3)=2 F(2), \\
& G(1)+G(3)>2 G(2), \\
& H(1)+H(3)<2 H(2) .
\end{aligned}
$$

Then it is easy to see that $F, G, H$ are representatives of the three $3: 2$ equivalence classes.

In Section 2 we shall obtain a formula for the number of $3: r$ equivalence classes for general $r$. To simplify discussions we shall consider only the "normalised" ranking functions which satisfy the extra conditions that $F(n)=0$ and $F(1)=1$. By Example 1.1 it is sufficient to consider only normalised functions.

EXAMPLE 1.3. For $n=3$ and $r=2$ a normalised ranking function $F$ is characterised by $F(2)$ which lies in the open interval $(0,1)$ and the equivalence classes are $\left(0, \frac{1}{2}\right),\left\{\frac{7}{2}\right\},\left(\frac{1}{2}, 1\right)$.

In general for $n \geq 3$ the normalised ranking functions are characterised by an open subset $S(n)$ of $\mathbf{R}^{n-2}$ corresponding to the possible values of $(F(2), F(3), \ldots, F(n-1))$. We associate with $S(n)$ the usual topology of $\mathbf{R}^{n-2}$ restricted to $S(n)$.

DEFINITION 1.4. We say that a normalised ranking function $F$ is $n: r$ stable if there exists an open set $U \subset S(n)$ such that $F \in U$ and $U$ is contained in the $n: r$ equivalence class containing $F$.

In other words there exists a neighbourhood $U$ of $F$ such that all ranking functions in $U$ always rank $n$ competitors who compete in $r$ races in exactly the same order as $F$.

EXAMPLE 1.5. Let $F, G, H$ be defined as in Example 1.2. Then we 
note that $G$ and $H$ are $3: 2$ stable and $F$ is not $3: 2$ stable.

It is easy to see that the $n: r$ stable normalised ranking functions are generic (that is they form an open dense set in $S(n)$ ). They are dense because the unstable functions lie on a finite set of hyperplanes which intersect $S(n)$.

EXAMPLE 1.6. Let $n=4, r=2, f(2)=x$, and $f(3)=y$. Then all the normalised ranking functions are in the open region bounded by the triangle with edges $x=y, x=1, y=0$. The complete set of results is $\{1,1\},\{1,2\},\{1,3\},\{1,4\},\{2,2\},\{2,3\},\{2,4\}$, $\{3,3\},\{3,4\},\{4,4\}$. We write down the pairs of results which are free to be ranked either way and the equation which gives them equal ranking:

$$
\begin{aligned}
& \{1,3\} \quad\{2,2\} \quad 1+y=2 x \text {, } \\
& \{1,4\} \quad\{2,2\} \quad 1=2 x \text {, } \\
& \{1,4\} \quad\{2,3\} \quad 1=x+y \text {, } \\
& \{1,4\} \quad\{3,3\} \quad I=2 y \text {, } \\
& \{2,4\} \quad\{3,3\} \quad x=2 y \text {. }
\end{aligned}
$$

Any ranking function which satisfies any one of these equations is not stable. From Figure 1 we see that there are ten equivalence classes of stable ranking functions.

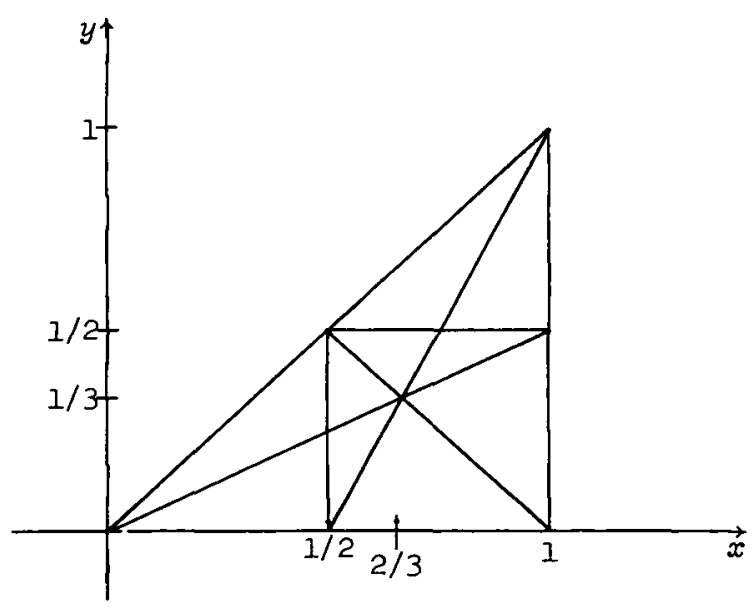

FIGURE 1 
For a fixed $n$, bifurcations of the equivalence classes occur as $r$ increases and we have two related problems. To determine the bifurcations and the number of equivalence classes for each fixed $r$. This problem is solved for $n=3$ in Section 2. It is interesting to note that the bifurcation set obtained (see Figure 2) is a ramified structure of the kind associated with a generalized catastrophe (see [1], p. 107). The corresponding problem for $n=4$ is more complex. Algebraic and combinatorial properties of ranking functions are studied in [2].

\section{The enumeration of $3: r$ ranking functions}

We let $\phi(h)$ denote Euler's $\phi$-function. That is $\phi(h)$ is the number of natural numbers less than or equal to $h$ relatively prime to $h$.

THEOREM 2.1. The number of $3: r$ equivalence classes of stable ranking functions is $\sum_{j=1}^{r} \phi(j)$.

Proof. Suppose two results $\left\{\alpha_{k}\right\}_{1 \leq k \leq r}$ and $\left\{\beta_{k}\right\}_{1 \leq k \leq r}$ have the following properties:

(a) no $\alpha_{k}$ is the same as a $\beta_{k}$;

(b) it is possible to rank $\left\{\alpha_{k}\right\}_{1 \leq k \leq r}$ above or below $\left\{\beta_{k}\right\}_{1 \leq k \leq r}$.

It can be seen that it is precisely such a pair of results which leads to the bifurcation of an equivalence class of ranking functions which are $3:(r-1)$ stable. Further it is clear that properties $(a)$ and $(b)$ can only be satisfied by the results $\{2,2, \ldots, 2\}$ and $\{1,1, \ldots, 1,3, \ldots, 3\}$ where the second result contains $q$ first places and $p-q$ third places. A ranking function $E$ which ranks these two results equal must satisfy the equation

$$
r F(2)=q F(1)+(r-q) F(3) .
$$

Since we need only consider normalised ranking functions with $F(1)=1$ and $F(3)=0$ it follows that $F(2)=q / r$. 


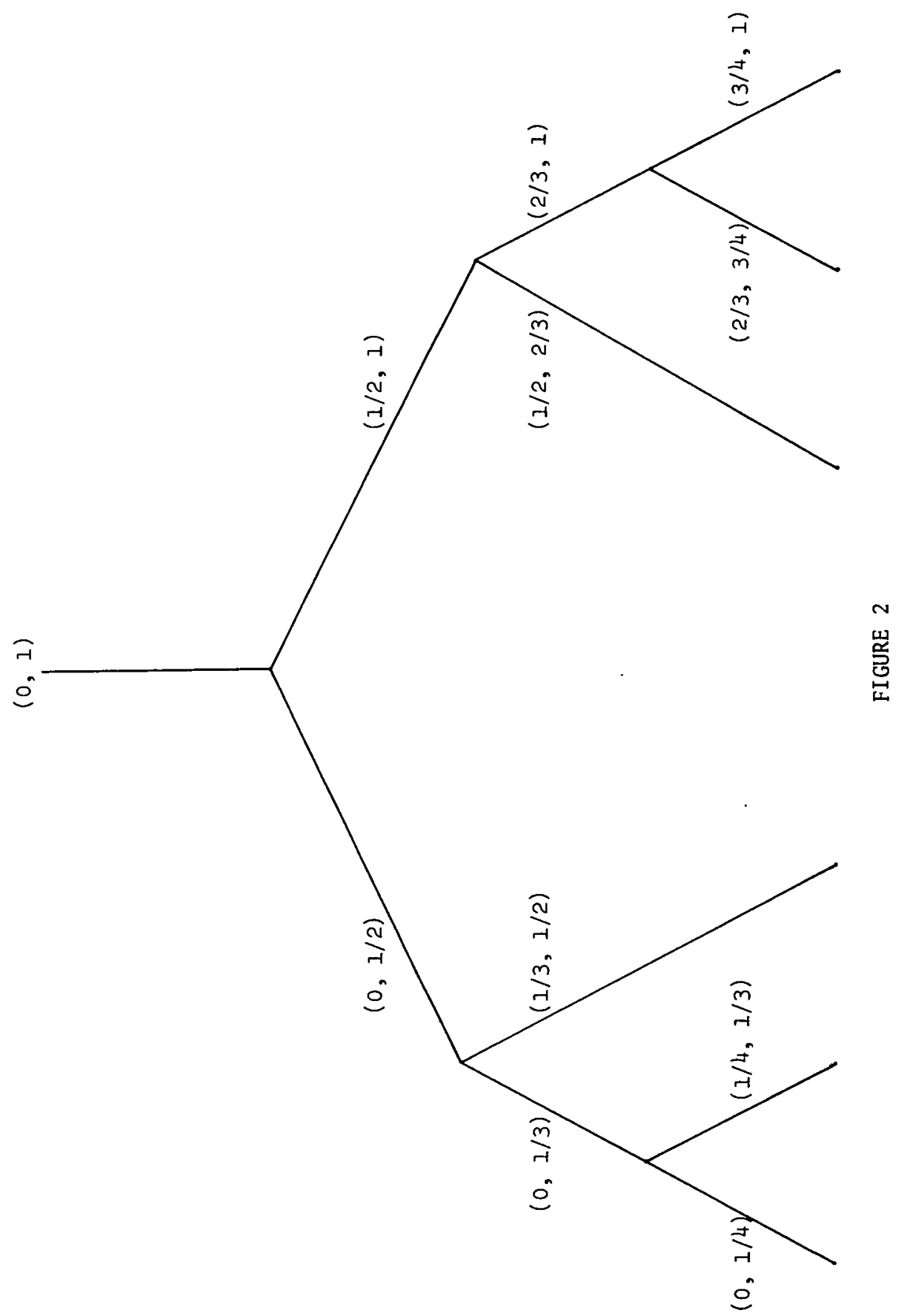


Hence the $3: r$ unstable normalised ranking functions are precisely those which satisfy the condition $F(2)=q / r$ where $1 \leq q \leq r-1$. A bifurcation occurs provided $q$ and $r$ are relatively prime. If $q$ and $r$ have a common factor the normalised ranking function $F$ which satisfies $F(2)=q / r$ has already become unstable at a smaller value of $r$. In Figure 2 we show the bifurcations of the equivalence classes of $3: r$ stable normalised ranking functions which occur at $r=2,3,4$.

- It is clear that the number of bifurcations for each $r$ is in fact $\phi(r)$. Also the number of $3: r$ unstable normalised ranking functions is $\sum_{j=2}^{r} \phi(j)$. The number of $3: r$ equivalence classes of stable ranking functions exceeds the number of $3: r$ unstable normalised ranking functions by one and hence can be written $\sum_{j=1}^{r} \phi(j)$. This completes the proof.

Corresponding results for $n>3$ are more complex. We note however that it is easy to prove that an equivalence class of stable ranking functions in $S(n)$ is convex.

\section{References}

[1] René Thom, Structural stability and morphogenesis: an outzine of a general theory of models (translated by D.H. Fowler. Benjamin, Reading, Massachusetts; London; Amsterdam; Don Mills, Ontario; Sydney; Tokyo; 1975).

[2] W.J. Walker, "Algebraic and combinatorial results for ranking competitors in a sequence of races", Discrete Math. 14 (1976), 297-304.

Department of Mathematics,

University of Auckland,

Auckl and,

New Zealand. 